CRYSTALLOGRAPHIC COMMUNICATIONS

ISSN 2056-9890

Received 24 September 2021

Accepted 1 October 2021

Edited by O. Blacque, University of Zürich, Switzerland

Keywords: crystal structure; organosiloxane; pyridoxal; Schiff base; macrocycle.

CCDC reference: 2113407

Supporting information: this article has supporting information at journals.iucr.org/e

\section{Formation of a macrocycle from dichlorodimethyl- silane and a pyridoxalimine Schiff base ligand}

\author{
Uwe Böhme, ${ }^{a *}$ Anke Schwarzer ${ }^{b}$ and Betty Günther ${ }^{a}$ \\ 'Institut für Anorganische Chemie, Technische Universität Bergakademie Freiberg, Leipziger Str. 29, 09599 Freiberg, \\ Germany, and ${ }^{\mathbf{b}}$ Institut für Organische Chemie, Technische Universität Bergakademie Freiberg, Leipziger Str. 29, 09599 \\ Freiberg, Germany. ${ }^{*}$ Correspondence e-mail: uwe.boehme@chemie.tu-freiberg.de
}

The reaction of dichlorodimethylsilane with a polydentate Schiff base ligand derived from pyridoxal and 2-ethanolamine yielded the macrocyclic silicon compound $(8 E, 22 E)-4,4,12,18,18,26$-hexamethyl-3,5,17,19-tetraoxa-8,13,22,27tetraaza-4,18-disilatricyclo[22.4.0.0 ${ }^{10,15}$ ]octacosa-1(24),8,10,12,14,22,25,27-octaene-11,25-diol, $\mathrm{C}_{24} \mathrm{H}_{36} \mathrm{~N}_{4} \mathrm{O}_{6} \mathrm{Si}_{2}$. The asymmetric unit contains the half macrocycle with an intramolecular $\mathrm{O}-\mathrm{H} \cdots \mathrm{N}$ hydrogen bond between the imine nitrogen atom and a neighbouring oxygen atom. The crystal structure is dominated by $\mathrm{C}-\mathrm{H} \cdots \mathrm{O}$ and $\mathrm{C}-\mathrm{H} \cdots \pi$ interactions, which form a high ordered molecular network.

\section{Chemical context}

The heterocyclic aldehyde pyridoxal is one of the active forms of vitamin $\mathrm{B}_{6}$. This vitamin is an essential cofactor to a large number of enzymes that catalyze many reactions of amino acids (Sykes et al., 1991). The coordination chemistry of Schiff bases generated from amino acids and pyridoxal with transition metal ions has been investigated intensive (Christensen, 1957; Long et al., 1980; Dawes et al., 1982; Walz et al., 1983; Rao et al., 1985; Astheimer et al., 1985; Sykes et al., 1991; Costa Pessoa et al., 1999). We are working on silicon complexes with tridentate $O, N, O$-ligands (Böhme \& Günther, 2007a; Böhme et al., 2006; Paul et al., 2014; Warncke et al., 2012; Schwarzer et $a l ., 2018)$. Therefore, we prepared a Schiff base from pyridoxal and 2-aminoethanol as a potential $O, N, O$-ligand. The crystal structure of this molecule, 4-[(2-hydroxyethyl)iminomethyl]-5hydroxymethyl-2-methylpyridine-3-ol (I), was published earlier (Böhme \& Günther, 2007b). Compound (I) was used recently as ligand molecule to coordinate copper and silver ions (Annaraj \& Neelakantan, 2014, 2015). Herein we report the results of reaction between (I) and dichlorodimethylsilane.

There are several potential coordination sites at the ligand

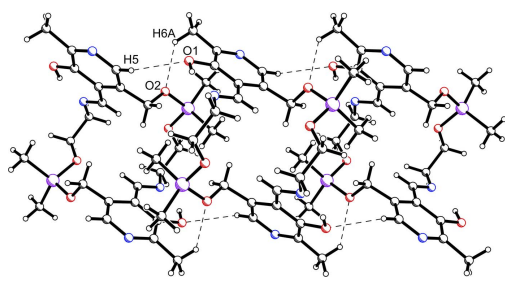
molecule (I): the pyridine and the imino nitrogen atoms, two aliphatic and one phenolic hydroxyl groups. The presence of these functional groups makes it difficult to predict the structure of the reaction product with dichlorodimethylsilane. It was our initial goal to prepare a pentacoordinate silicon complex like (II). Surprisingly the macrocyclic silicon compound (III) was obtained from the reaction of (I) with $\mathrm{Me}_{2} \mathrm{SiCl}_{2}$. The reaction was performed in tetrahydrofuran in presence of triethylamine as supporting base to remove the hydrogen chloride, which is formed during the reaction. Recrystallization of the raw product from 1,2-dimethoxyethane and diethyl ether gave yellow crystals suitable for structure analysis. 


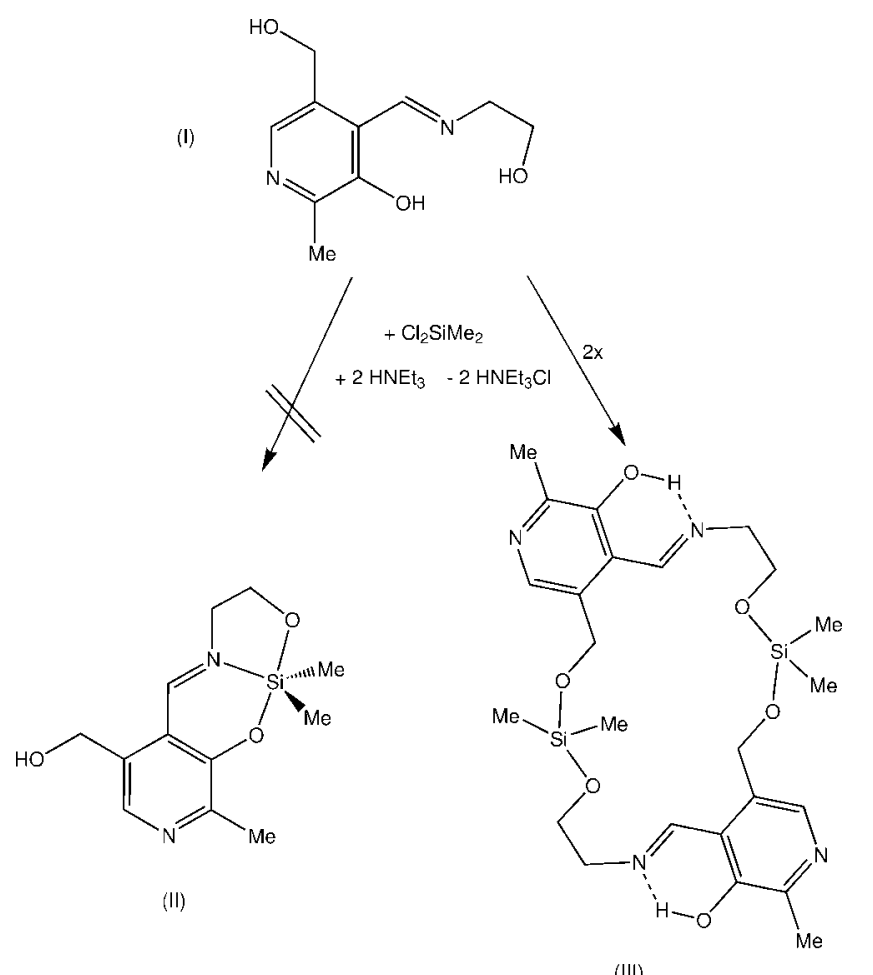

(III)

\section{Structural commentary}

Compound (III) crystallizes in the monoclinic space group $12 / \mathrm{c}$ with the half macrocycle in the asymmetric unit. Fig. 1 shows the asymmetric unit and the atomic labelling scheme. The

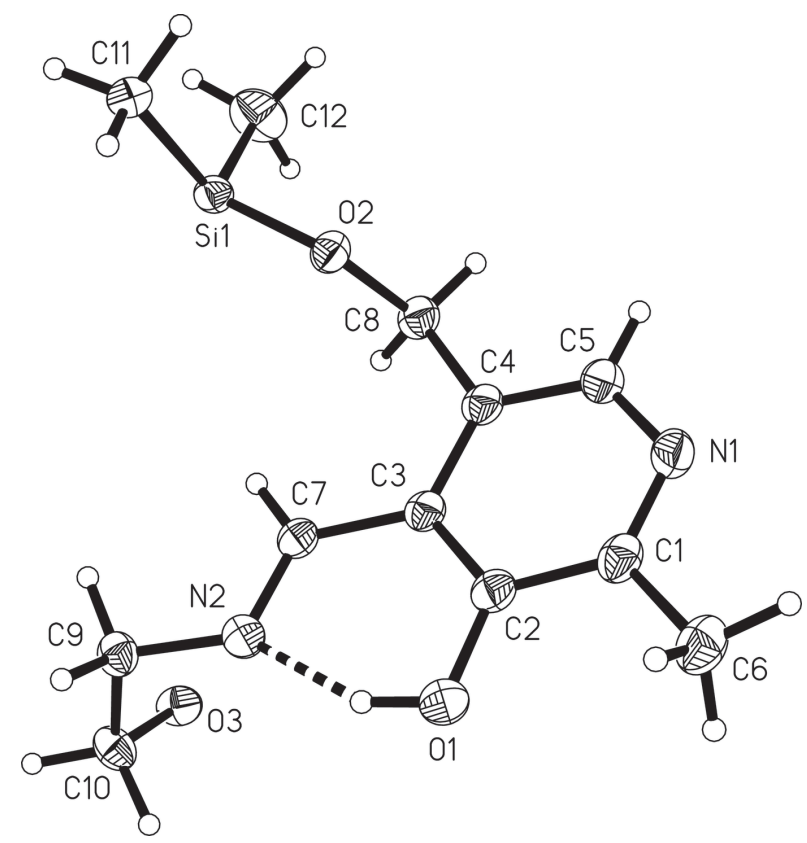

Figure 1

The asymmetric unit of (III), drawn with $50 \%$ probability displacement ellipsoids. The dashed line shows the intramolecular $\mathrm{O} 1-\mathrm{H} 1 \cdots \mathrm{N} 2$ hydrogen bond.
Table 1

Selected geometric parameters $\left(\AA,^{\circ}\right)$.

\begin{tabular}{lrlr}
\hline $\mathrm{Si} 1-\mathrm{O} 2$ & $1.6435(9)$ & $\mathrm{Si} 1-\mathrm{C} 12$ & $1.8443(14)$ \\
$\mathrm{Si} 1-3^{\mathrm{i}}$ & $1.6487(9)$ & $\mathrm{Si} 1-\mathrm{C} 11$ & $1.8589(15)$ \\
& & & \\
$\mathrm{O} 2-\mathrm{Si} 1-\mathrm{O} 3^{\mathrm{i}}$ & $103.40(5)$ & $\mathrm{O}^{\mathrm{i}}-\mathrm{Si} 1-\mathrm{C} 11$ & $109.52(6)$ \\
$\mathrm{O} 2-\mathrm{Si} 1-\mathrm{C} 12$ & $106.94(6)$ & $\mathrm{C} 12-\mathrm{Si} 1-\mathrm{C} 11$ & $113.16(7)$ \\
$\mathrm{O}^{\mathrm{i}}-\mathrm{Si} 1-\mathrm{C} 12$ & $112.06(6)$ & $\mathrm{C} 8-\mathrm{O} 2-\mathrm{Si} 1$ & $123.61(8)$ \\
$\mathrm{O} 2-\mathrm{Si} 1-\mathrm{C} 11$ & $111.33(7)$ & $\mathrm{C} 10-\mathrm{O} 3-\mathrm{Si1}^{\mathrm{i}}$ & $123.50(8)$ \\
\hline
\end{tabular}

Symmetry code: (i) $-x+2, y,-z+\frac{1}{2}$.

macrocycle is generated by a crystallographic $C 2$ axis through the centre of the macrocycle (Fig. 2). The silicon atom is bound to the two methyl groups and to the aliphatic oxygen atoms $\mathrm{O} 2$ and $\mathrm{O} 3$, thus forming a macrocycle (Fig. 2). A quite similar macrocycle has been obtained from the reaction of a related pyridoxal-derived Schiff base and dichlorodiphenylsilane (Böhme et al., 2008). The short $\mathrm{Si}-\mathrm{O}$ bonds (see Table 1) are in the range for comparable $\mathrm{Si}-\mathrm{O}$ bonds (Wagler et al., 2005; Böhme et al., 2006, 2008; Böhme \& Günther, 2007a; Böhme \& Foehn, 2007). The silicon atom is distorted tetrahedral with bond angles between 103.40 (5) and $113.16(7)^{\circ}$ (Table 1). The rather large bond angles at the oxygen atoms (see Table 1) have been explained by the ionic character of the $\mathrm{Si}-\mathrm{O}$ bonds (Gillespie \& Johnson, 1997). There is a strong intramolecular $\mathrm{O}-\mathrm{H} \cdots \mathrm{N}$ interaction (entry 1, Table 2) between the imine nitrogen atom $\mathrm{N} 2$ and the $\mathrm{O} 1-\mathrm{H} 1$ group in the neighbouring position at the pyridoxal ring. The formation of hydrogen bridges between the imine nitrogen atom and an ortho-hydroxyl group is a feature that is often observed in Schiff bases with $o$-hydroxy groups (Hökelek et al., 2004; Filarowski et al., 1999). This strong intramolecular $\mathrm{O}-\mathrm{H} \cdots \mathrm{N}$ interaction leads to a six-membered pseudo ring consisting of $\mathrm{H} 1-\mathrm{O} 1-\mathrm{C} 2-\mathrm{C} 3-\mathrm{C} 7-\mathrm{N} 2$. This pseudo ring is planar with an r.m.s. deviation of $0.009 \AA$ from the ring plane. According to the graph-set notation proposed by Etter et al. (1990), these

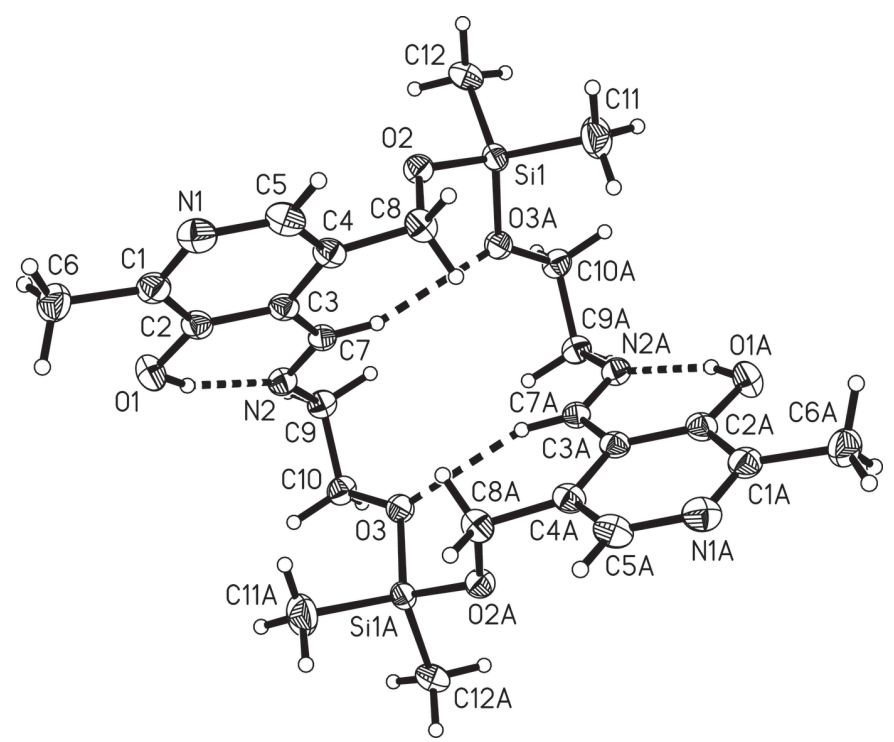

Figure 2

The molecular structure of (III), drawn with $50 \%$ probability displacement ellipsoids. 
Table 2

Hydrogen-bond geometry $\left(\AA,^{\circ}\right)$.

$C g 1$ is the centroid of the N1/C1-C5 ring.

\begin{tabular}{lllll}
\hline$D-\mathrm{H} \cdots A$ & $D-\mathrm{H}$ & $\mathrm{H} \cdots A$ & $D \cdots A$ & $D-\mathrm{H} \cdots A$ \\
\hline $\mathrm{O} 1-\mathrm{H} 1 \cdots \mathrm{N} 2$ & $0.90(2)$ & $1.76(2)$ & $2.5923(15)$ & $153.2(18)$ \\
$\mathrm{C} 5-\mathrm{H} 5 \cdots \mathrm{O} 1^{\text {ii }}$ & 0.95 & 2.69 & $3.5451(16)$ & 151 \\
$\mathrm{C} 6-\mathrm{H} 6 A \cdots \mathrm{O} 2^{\text {iii }}$ & 0.98 & 2.59 & $3.3464(17)$ & 134 \\
$\mathrm{C} 7-\mathrm{H} 7 \cdots \mathrm{O} 3^{\mathrm{i}}$ & 0.95 & 2.57 & $3.4882(15)$ & 162 \\
$\mathrm{C} 9-\mathrm{H} 9 B \cdots \mathrm{O} 2^{\text {iv }}$ & 0.99 & 2.60 & $3.5087(16)$ & 153 \\
$\mathrm{C} 9-\mathrm{H} 9 B \cdots C g 1^{\text {iv }}$ & 0.99 & 3.31 & $4.039(2)$ & 131 \\
$\mathrm{C} 11-\mathrm{H} 11 A \cdots C g 1^{\text {ii }}$ & 0.98 & 2.85 & $3.7880(2)$ & 160
\end{tabular}

Symmetry codes: (i) $-x+2, y,-z+\frac{1}{2}$; (ii) $x,-y+1, z-\frac{1}{2}$; (iii) $x,-y+1, z+\frac{1}{2}$; (iv) $-x+\frac{3}{2},-y+\frac{1}{2},-z+\frac{1}{2}$.

hydrogen bonds form motifs with an $S_{1}^{1}(6)$ graph-set descriptor. The hydrogen bonds $\mathrm{C} 7-\mathrm{H} 7$. O 3 link different parts within one macrocycle via intra-annular hydrogen bonds (Fig. 2).

\section{Supramolecular features}

A bifurcated intermolecular $\mathrm{C}-\mathrm{H} \cdots \mathrm{O}$ interaction is observed at $\mathrm{O} 2$ (Table 2). The interaction of $\mathrm{C} 6-\mathrm{H} 6 A \cdots \mathrm{O} 2$ and $\mathrm{C} 5-\mathrm{H} 5 \cdots \mathrm{O} 1$ results in a chain along the crystallographic $b$-axis. The $\mathrm{C}-\mathrm{H} \cdots \mathrm{O}$ interaction of $\mathrm{C} 9-\mathrm{H} 9 B$ with $\mathrm{O} 2$ connects adjacent chains (Fig. 3).

Apart from the relevant $\mathrm{C}-\mathrm{H} \cdots \mathrm{O}$ interaction, two $\mathrm{C}-$ $\mathrm{H} \cdots \pi$ contacts with the pyridine moiety $(C g 1)$ are observed. First, a bifurcation at $\mathrm{H} 9 B(d=3.31 \AA)$ shows up within the $\mathrm{C}-\mathrm{H} \cdots \mathrm{O}$ chains along the $c$ axis. Furthermore, C11$\mathrm{H} 11 A \cdots C g 1(d=2.85 \AA)$ supports the $\mathrm{C}-\mathrm{H} \cdots \mathrm{O}$ interactions of $\mathrm{H} 5$ and $\mathrm{H} 6 A$.

In summary, the crystal structure is dominated by $\mathrm{C}-$ $\mathrm{H} \cdots \mathrm{O}$ and $\mathrm{C}-\mathrm{H} \cdots \pi$ interactions, forming a highly ordered molecular network.

The potential bonding sites in combination with the cavity of the macrocycle makes (III) a suitable candidate for supramolecular recognition processes. The available pyridine $\mathrm{N}$, azomethine $\mathrm{N}$, and $\mathrm{OH}$ groups could be useful for the generation of nanostructures via complexation with transition metals (Leininger et al., 2000).

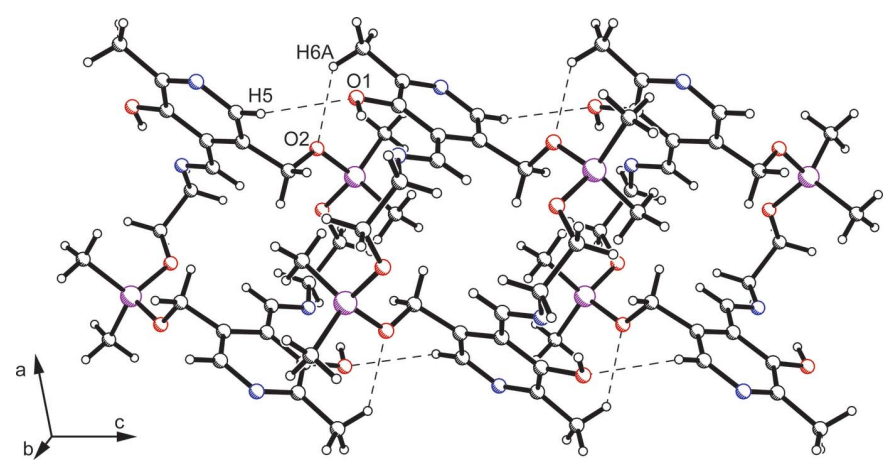

Figure 3

Packing excerpt of (III) showing $\mathrm{C}-\mathrm{H} \cdots \mathrm{O}$ hydrogen bonds (dashed lines).

\section{Database survey}

A CSD search with ConQuest (Bruno et al., 2002) for macrocycles containing Schiff bases from pyridoxal and 2-aminoalcohols showed that only one comparable silicon compound exists (Böhme et al., 2008, refcode MOKVEO). The main differences between these two structures of siliconcontaining macrocycles are as follows. First, (III) was found to crystallize without solvent while MOKVEO encloses chlorofom molecules. Probably as a result, the symmetry is lower in MOKVEO (triclinic, $P \overline{\overline{1}}$ ) than in (III) showing the monoclinic $I 2 / c$ symmetry. On the basis of the structure of (III) presented here and the former investigation (Böhme et al., 2008), it can be assumed that pyridoxalimine-derived Schiff bases prefer the formation of macrocycles with diorganosilane units. However, it seems to be possible that compound (I) can also act as a tridentate $O, N, O$-ligand, as was shown recently with a hexacoordinate titanium complex (Böhme \& Günther, 2020).

\section{Synthesis and crystallization}

The preparation of (III) was performed in Schlenk tubes under argon with dry and air-free solvents.

Compound (III) was prepared by reaction of 4-[(2-hydroxyethyl)iminomethyl]-5-hydroxymethyl-2-methylpyridine3-ol (I) (1.7 g, $8 \mathrm{mmol})$ with dichlorodimethylsilane $(1.03 \mathrm{~g}$, $8 \mathrm{mmol})$ in the presence of triethylamine $(2.02 \mathrm{~g}, 20 \mathrm{mmol})$. The reaction was performed in dry tetrahydrofuran at room temperature. A white precipitate of triethylamine hydrochloride formed upon stirring of the mixture for five days. After this period, the triethylamine hydrochloride was filtered off and washed with tetrahydrofuran. The solvent was removed in vacuo from the resulting clear yellow solution. The remaining solid was extracted with 1,2-dimethoxyethane. Addition of diethyl ether and cooling to $278 \mathrm{~K}$ yielded yellow crystals of (III) (1.66 g, 78\%, m.p. 390 K).

NMR $\left(\mathrm{CDCl}_{3}, 300 \mathrm{~K}\right.$, TMS, in p.p.m.): ${ }^{29} \mathrm{Si}:-0.1 .{ }^{1} \mathrm{H}: \delta=$ $0.14\left(s, \mathrm{Me}_{2} \mathrm{Si}, 6 \mathrm{H}\right), 2.50\left(s, \mathrm{CH}_{3}\right.$ pyridoxal, $\left.3 \mathrm{H}\right), 3.71,3.90(t$, $\left.\mathrm{N}-\mathrm{CH}_{2}-\mathrm{CH}_{2}-\mathrm{O}, 4 \mathrm{H}\right), 4.78\left(s, \mathrm{CH}_{2}-\mathrm{O}\right.$ pyridoxal, $\left.2 \mathrm{H}\right), 7.89$ $(s, \mathrm{CH}$ pyridoxal, $1 \mathrm{H}), 8.84(s, \mathrm{HC}=\mathrm{N}, 1 \mathrm{H}), 14.05(s, \mathrm{OH}$ pyridoxal, $1 \mathrm{H}) .{ }^{13} \mathrm{C}: 3.0\left(\mathrm{Me}_{2} \mathrm{Si}\right), 22.0\left(\mathrm{CH}_{3}\right.$ pyridoxal $), 63.3$, $64.6\left(\mathrm{~N}-\mathrm{CH}_{2}-\mathrm{CH}_{2}-\mathrm{O}\right), 66.4\left(\mathrm{CH}_{2}-\mathrm{O}\right.$ pyridoxal), 122.6, 133.4, 140.8, 153.8, 157.8 (five C pyridoxal), $167.5(\mathrm{HC}=\mathrm{N})$.

\section{Refinement}

Crystal data, data collection and structure refinement details are summarized in Table 3. The hydrogen atom at $\mathrm{O} 1$ was refined freely. The methyl groups were refined as idealized rigid groups allowed to rotate but not tip (AFIX 137; $\mathrm{C}-\mathrm{H}=$ $\left.0.98 \AA, \mathrm{H}-\mathrm{C}-\mathrm{H}=109.5^{\circ}\right)$. Other hydrogens were included using a riding model starting from calculated positions $(\mathrm{C}-$ $\left.\mathrm{H}_{\text {aromatic }}=0.95, \mathrm{C}-\mathrm{H}_{\text {methylene }}=0.99 \AA\right)$. The $U_{\text {iso }}(\mathrm{H})$ values were fixed at 1.5 (for the methyl $\mathrm{H}$ ) or 1.2 times the equivalent $U_{\text {eq }}$ value of the parent carbon atoms. 


\section{Funding information}

Funding for this research was provided by: Open Access Funding by the Publication Fund of the TU Bergakademie Freiberg .

\section{References}

Annaraj, B. \& Neelakantan, M. A. (2014). Anal. Methods 6, 96109615.

Annaraj, B. \& Neelakantan, M. A. (2015). Eur. J. Med. Chem. 102,18.

Astheimer, H., Nepveu, F., Walz, L. \& Haase, W. (1985). J. Chem. Soc. Dalton Trans. pp. 315-320.

Böhme, U. \& Foehn, I. C. (2007). Acta Cryst. C63, o613-o616.

Böhme, U. \& Günther, B. (2007a). Inorg. Chem. Commun. 10, 482484.

Böhme, U. \& Günther, B. (2007b). Acta Cryst. C63, o641-o642.

Böhme, U. \& Günther, B. (2020). CSD Communication (CCDC No. 2048270). CCDC, Cambridge, England. https://dx.doi.org/10.5517/ ccdc.csd.cc26rd7m

Böhme, U., Günther, B. \& Schwarzer, A. (2008). Acta Cryst. C64, o630-o632.

Böhme, U., Wiesner, S. \& Günther, B. (2006). Inorg. Chem. Commun. 9, 806-809.

Bruno, I. J., Cole, J. C., Edgington, P. R., Kessler, M., Macrae, C. F., McCabe, P., Pearson, J. \& Taylor, R. (2002). Acta Cryst. B58, 389397.

Christensen, H. N. (1957). J. Am. Chem. Soc. 79, 4073-4078.

Costa Pessoa, J., Cavaco, I., Correia, I., Duarte, M. T., Gillard, R. D., Henriques, R. T., Higes, F. J., Madeira, C. \& Tomaz, I. (1999). Inorg. Chim. Acta, 293, 1-11.

Dawes, H. M., Waters, J. M. \& Waters, T. N. (1982). Inorg. Chim. Acta, 66, 29-36.

Etter, M. C., MacDonald, J. C. \& Bernstein, J. (1990). Acta Cryst. B46, 256-262.

Farrugia, L. J. (2012). J. Appl. Cryst. 45, 849-854.

Filarowski, A., Głowiaka, T. \& Koll, A. (1999). J. Mol. Struct. 484, 7589.

Gillespie, R. J. \& Johnson, S. A. (1997). Inorg. Chem. 36, 3031-3039.

Hökelek, T., Bilge, S., Demiriz, Ş., Özgüç, B. \& Kılıç, Z. (2004). Acta Cryst. C60, o803-o805.

Leininger, S., Olenyuk, B. \& Stang, P. J. (2000). Chem. Rev. 100, 853908.

Long, G. J., Wrobleski, J. T., Thundathil, R. V., Sparlin, D. M. \& Schlemper, E. O. (1980). J. Am. Chem. Soc. 102, 6040-6046.

Paul, L. E. H., Foehn, I. C., Schwarzer, A., Brendler, E. \& Böhme, U. (2014). Inorg. Chim. Acta, 423, 268-280.

Rao, S. P. S., Manohar, H. \& Bau, R. (1985). J. Chem. Soc. Dalton Trans. pp. 2051-2057.
Table 3

Experimental details.

\begin{tabular}{|c|c|}
\hline \multicolumn{2}{|l|}{ Crystal data } \\
\hline Chemical formula & $\mathrm{C}_{24} \mathrm{H}_{36} \mathrm{~N}_{4} \mathrm{O}_{6} \mathrm{Si}_{2}$ \\
\hline$M_{\mathrm{r}}$ & 532.75 \\
\hline Crystal system, space group & Monoclinic, $I 2 / c$ \\
\hline Temperature $(\mathrm{K})$ & 153 \\
\hline$a, b, c(\AA)$ & $\begin{array}{l}12.9641(8), 16.8966(7) \\
13.1085(8)\end{array}$ \\
\hline$\beta\left({ }^{\circ}\right)$ & $101.198(5)$ \\
\hline$V\left(\AA^{3}\right)$ & $2816.7(3)$ \\
\hline$Z$ & 4 \\
\hline Radiation type & Мо $K \alpha$ \\
\hline$\mu\left(\mathrm{mm}^{-1}\right)$ & 0.17 \\
\hline Crystal size $(\mathrm{mm})$ & $0.40 \times 0.33 \times 0.15$ \\
\hline \multicolumn{2}{|l|}{ Data collection } \\
\hline Diffractometer & Stoe IPDS $2 \mathrm{~T}$ \\
\hline Absorption correction & Integration $(X-R E D ;$ Stoe, 2009) \\
\hline$T_{\min }, T_{\max }$ & $0.907,0.993$ \\
\hline $\begin{array}{l}\text { No. of measured, independent and } \\
\text { observed }[I>2 \sigma(I)] \text { reflections }\end{array}$ & $19293,3242,2833$ \\
\hline$R_{\text {int }}$ & 0.039 \\
\hline$(\sin \theta / \lambda)_{\max }\left(\AA^{-1}\right)$ & 0.650 \\
\hline \multicolumn{2}{|l|}{ Refinement } \\
\hline$R\left[F^{2}>2 \sigma\left(F^{2}\right)\right], w R\left(F^{2}\right), S$ & $0.032,0.082,1.08$ \\
\hline No. of reflections & 3242 \\
\hline No. of parameters & 169 \\
\hline $\mathrm{H}$-atom treatment & $\begin{array}{l}\mathrm{H} \text { atoms treated by a mixture of } \\
\text { independent and constrained } \\
\text { refinement }\end{array}$ \\
\hline$\Delta \rho_{\max }, \Delta \rho_{\min }\left(\mathrm{e} \AA^{-3}\right)$ & $0.32,-0.23$ \\
\hline
\end{tabular}

Computer programs: $X$-AREA and X-RED (Stoe, 2009), SHELXS (Sheldrick, 2008), SHELXL2017/1 (Sheldrick, 2015) and ORTEP-3 for Windows (Farrugia, 2012).

Schwarzer, S., Böhme, U., Fels, S., Günther, B. \& Brendler, E. (2018). Inorg. Chim. Acta, 483, 136-147.

Sheldrick, G. M. (2008). Acta Cryst. A64, 112-122.

Sheldrick, G. M. (2015). Acta Cryst. C71, 3-8.

Stoe (2009). $X$-RED and $X$-AREA. Stoe \& Cie, Darmstadt, Germany.

Sykes, A. G., Larsen, R. D., Fischer, J. R. \& Abbott, E. H. (1991). Inorg. Chem. 30, 2911-2916.

Wagler, J., Böhme, U., Brendler, E., Thomas, B., Goutal, S., Mayr, H., Kempf, B., Remennikov, G. Y. \& Roewer, G. (2005). Inorg. Chim. Acta, 358, 4270-4286.

Walz, L., Paulus, H., Haase, W., Langhof, H. \& Nepveu, F. (1983). J. Chem. Soc. Dalton Trans. pp. 657-664.

Warncke, G., Böhme, U., Günther, B. \& Kronstein, M. (2012). Polyhedron, 47, 46-52. 


\section{supporting information}

Acta Cryst. (2021). E77, 1099-1102［https://doi.org/10.1107/S2056989021010185]

Formation of a macrocycle from dichlorodimethylsilane and a pyridoxalimine Schiff base ligand

\section{Uwe Böhme, Anke Schwarzer and Betty Günther}

Computing details

Data collection: $X$-AREA (Stoe, 2009); cell refinement: $X$-AREA (Stoe, 2009); data reduction: $X$-RED (Stoe, 2009); program(s) used to solve structure: SHELXS (Sheldrick, 2008); program(s) used to refine structure: SHELXL2017/1 (Sheldrick, 2015); molecular graphics: ORTEP-3 for Windows (Farrugia, 2012); software used to prepare material for publication: SHELXL2017/1 (Sheldrick, 2015).

(8E,22E)-4,4,12,18,18,26-Hexamethyl-3,5,17,19-tetraoxa-8,13,22,27-tetraaza-4,18-

disilatricyclo[22.4.0.0 $0^{10,15}$ octacosa-1(24),8,10,12,14,22,25,27-octaene-11,25-diol

Crystal data

$\mathrm{C}_{24} \mathrm{H}_{36} \mathrm{~N}_{4} \mathrm{O}_{6} \mathrm{Si}_{2}$

$M_{r}=532.75$

Monoclinic, $I 2 / c$

$a=12.9641(8) \AA$

$b=16.8966(7) \AA$

$c=13.1085(8) \AA$

$\beta=101.198(5)^{\circ}$

$V=2816.7(3) \AA^{3}$

$Z=4$

Data collection

Stoe IPDS 2T

diffractometer

Radiation source: sealed X-ray tube, 12 x 0.4 $\mathrm{mm}$ long-fine focus

Plane graphite monochromator

Detector resolution: 6.67 pixels $\mathrm{mm}^{-1}$

rotation method scans

Absorption correction: integration

(X-RED; Stoe, 2009)

Refinement

Refinement on $F^{2}$

Least-squares matrix: full

$R\left[F^{2}>2 \sigma\left(F^{2}\right)\right]=0.032$

$w R\left(F^{2}\right)=0.082$

$S=1.08$

3242 reflections

169 parameters

0 restraints
$F(000)=1136$

$D_{\mathrm{x}}=1.256 \mathrm{Mg} \mathrm{m}^{-3}$

Mo $K \alpha$ radiation, $\lambda=0.71073 \AA$

Cell parameters from 19293 reflections

$\theta=3.2-28.8^{\circ}$

$\mu=0.17 \mathrm{~mm}^{-1}$

$T=153 \mathrm{~K}$

Prism, yellow

$0.40 \times 0.33 \times 0.15 \mathrm{~mm}$

$T_{\min }=0.907, T_{\max }=0.993$

19293 measured reflections

3242 independent reflections

2833 reflections with $I>2 \sigma(I)$

$R_{\text {int }}=0.039$

$\theta_{\max }=27.5^{\circ}, \theta_{\min }=2.0^{\circ}$

$h=-16 \rightarrow 16$

$k=-21 \rightarrow 21$

$l=-16 \rightarrow 16$

Hydrogen site location: mixed

$\mathrm{H}$ atoms treated by a mixture of independent and constrained refinement

$w=1 /\left[\sigma^{2}\left(F_{\mathrm{o}}^{2}\right)+(0.0343 P)^{2}+2.1897 P\right]$

where $P=\left(F_{\mathrm{o}}{ }^{2}+2 F_{\mathrm{c}}{ }^{2}\right) / 3$

$(\Delta / \sigma)_{\max }=0.001$

$\Delta \rho_{\max }=0.32 \mathrm{e} \AA^{-3}$

$\Delta \rho_{\min }=-0.23 \mathrm{e}^{-3}$ 


\section{Special details}

Geometry. All esds (except the esd in the dihedral angle between two 1.s. planes) are estimated using the full covariance matrix. The cell esds are taken into account individually in the estimation of esds in distances, angles and torsion angles; correlations between esds in cell parameters are only used when they are defined by crystal symmetry. An approximate (isotropic) treatment of cell esds is used for estimating esds involving l.s. planes.

Fractional atomic coordinates and isotropic or equivalent isotropic displacement parameters $\left(\AA^{2}\right)$

\begin{tabular}{|c|c|c|c|c|}
\hline & $x$ & $y$ & $z$ & $U_{\text {iso }} * / U_{\text {eq }}$ \\
\hline Sil & $0.86455(3)$ & $0.32645(2)$ & $-0.02263(2)$ & $0.02075(10)$ \\
\hline $\mathrm{O} 1$ & $0.71097(8)$ & $0.39251(6)$ & $0.42399(7)$ & $0.0296(2)$ \\
\hline H1 & $0.7514(16)$ & $0.3499(12)$ & $0.4190(14)$ & $0.044^{*}$ \\
\hline $\mathrm{O} 2$ & $0.80401(7)$ & $0.38781(5)$ & $0.04330(7)$ & $0.02281(19)$ \\
\hline $\mathrm{O} 3$ & $1.06149(7)$ & $0.27321(5)$ & $0.43122(7)$ & $0.02520(19)$ \\
\hline N1 & $0.67254(9)$ & $0.55915(7)$ & $0.24920(9)$ & $0.0294(2)$ \\
\hline $\mathrm{N} 2$ & $0.83696(8)$ & $0.29351(6)$ & $0.35941(8)$ & $0.0230(2)$ \\
\hline $\mathrm{C} 1$ & $0.66458(10)$ & $0.51009(8)$ & $0.32707(10)$ & $0.0259(3)$ \\
\hline $\mathrm{C} 2$ & $0.72289(10)$ & $0.43890(7)$ & 0.34287 (9) & $0.0233(2)$ \\
\hline $\mathrm{C} 3$ & $0.78833(9)$ & $0.41735(7)$ & $0.27376(9)$ & 0.0209 (2) \\
\hline $\mathrm{C} 4$ & $0.79399(10)$ & $0.46943(7)$ & $0.19053(9)$ & $0.0229(2)$ \\
\hline $\mathrm{C} 5$ & $0.73609(11)$ & $0.53867(8)$ & $0.18313(10)$ & $0.0282(3)$ \\
\hline H5 & 0.741329 & 0.574080 & 0.128082 & $0.034 *$ \\
\hline C6 & 0.58959 (11) & $0.53139(9)$ & $0.39701(11)$ & 0.0338 (3) \\
\hline H6A & 0.629045 & 0.540267 & 0.467780 & $0.051 *$ \\
\hline H6B & 0.539444 & 0.488047 & 0.397491 & $0.051^{*}$ \\
\hline $\mathrm{H} 6 \mathrm{C}$ & 0.551402 & 0.579715 & 0.371352 & $0.051^{*}$ \\
\hline $\mathrm{C} 7$ & $0.84345(9)$ & $0.34072(7)$ & $0.28447(9)$ & $0.0206(2)$ \\
\hline H7 & 0.884566 & 0.326031 & 0.234897 & $0.025^{*}$ \\
\hline $\mathrm{C} 8$ & $0.85625(10)$ & $0.45009(7)$ & $0.10775(10)$ & $0.0252(3)$ \\
\hline H8A & 0.861902 & 0.497621 & 0.065010 & $0.030 *$ \\
\hline H8B & 0.928130 & 0.433117 & 0.140566 & $0.030 *$ \\
\hline C9 & $0.89177(10)$ & $0.21754(7)$ & $0.36341(10)$ & $0.0236(2)$ \\
\hline H9A & 0.914711 & 0.208345 & 0.296627 & $0.028^{*}$ \\
\hline H9B & 0.843029 & 0.174374 & 0.373348 & $0.028 *$ \\
\hline $\mathrm{C} 10$ & $0.98674(10)$ & $0.21622(7)$ & $0.45164(10)$ & $0.0248(3)$ \\
\hline $\mathrm{H} 10 \mathrm{~A}$ & 0.964942 & 0.228849 & 0.518104 & $0.030 *$ \\
\hline H10B & 1.018780 & 0.162825 & 0.457622 & $0.030 *$ \\
\hline $\mathrm{C} 11$ & $0.94958(13)$ & $0.38050(10)$ & $-0.09824(13)$ & $0.0406(4)$ \\
\hline H11A & 0.907504 & 0.419996 & -0.142798 & $0.061 *$ \\
\hline H11B & 0.979557 & 0.342955 & -0.141483 & $0.061^{*}$ \\
\hline $\mathrm{H} 11 \mathrm{C}$ & 1.006491 & 0.407029 & -0.050283 & $0.061 *$ \\
\hline $\mathrm{C} 12$ & $0.76208(11)$ & $0.26635(8)$ & $-0.10486(10)$ & $0.0284(3)$ \\
\hline $\mathrm{H} 12 \mathrm{~A}$ & 0.717809 & 0.241394 & -0.061306 & $0.043^{*}$ \\
\hline H12B & 0.795342 & 0.225328 & -0.140318 & $0.043^{*}$ \\
\hline $\mathrm{H} 12 \mathrm{C}$ & 0.718640 & 0.300404 & -0.156636 & $0.043 *$ \\
\hline
\end{tabular}


Atomic displacement parameters $\left(\AA^{2}\right)$

\begin{tabular}{lllllll}
\hline & $U^{11}$ & $U^{22}$ & $U^{33}$ & $U^{12}$ & $U^{13}$ & $U^{23}$ \\
\hline Si1 & $0.02231(17)$ & $0.02191(17)$ & $0.01900(16)$ & $0.00084(12)$ & $0.00636(12)$ & $0.00114(12)$ \\
O1 & $0.0360(5)$ & $0.0297(5)$ & $0.0259(4)$ & $0.0028(4)$ & $0.0128(4)$ & $0.0013(4)$ \\
O2 & $0.0232(4)$ & $0.0221(4)$ & $0.0228(4)$ & $-0.0002(3)$ & $0.0034(3)$ & $-0.0036(3)$ \\
O3 & $0.0241(4)$ & $0.0287(5)$ & $0.0230(4)$ & $-0.0076(4)$ & $0.0054(3)$ & $-0.0008(3)$ \\
N1 & $0.0338(6)$ & $0.0237(5)$ & $0.0288(5)$ & $0.0025(4)$ & $0.0011(4)$ & $-0.0044(4)$ \\
N2 & $0.0219(5)$ & $0.0229(5)$ & $0.0237(5)$ & $-0.0010(4)$ & $0.0029(4)$ & $0.0000(4)$ \\
C1 & $0.0251(6)$ & $0.0259(6)$ & $0.0254(6)$ & $-0.0010(5)$ & $0.0012(5)$ & $-0.0074(5)$ \\
C2 & $0.0240(6)$ & $0.0240(6)$ & $0.0209(5)$ & $-0.0033(5)$ & $0.0020(4)$ & $-0.0039(4)$ \\
C3 & $0.0214(5)$ & $0.0206(5)$ & $0.0195(5)$ & $-0.0040(4)$ & $0.0011(4)$ & $-0.0031(4)$ \\
C4 & $0.0264(6)$ & $0.0203(6)$ & $0.0211(5)$ & $-0.0049(4)$ & $0.0026(4)$ & $-0.0033(4)$ \\
C5 & $0.0374(7)$ & $0.0213(6)$ & $0.0246(6)$ & $-0.0017(5)$ & $0.0026(5)$ & $-0.0019(5)$ \\
C6 & $0.0283(7)$ & $0.0370(7)$ & $0.0363(7)$ & $0.0046(6)$ & $0.0072(5)$ & $-0.0080(6)$ \\
C7 & $0.0200(5)$ & $0.0218(6)$ & $0.0196(5)$ & $-0.0030(4)$ & $0.0024(4)$ & $-0.0033(4)$ \\
C8 & $0.0306(6)$ & $0.0210(6)$ & $0.0246(6)$ & $-0.0058(5)$ & $0.0071(5)$ & $-0.0014(4)$ \\
C9 & $0.0237(6)$ & $0.0203(6)$ & $0.0263(6)$ & $-0.0027(4)$ & $0.0034(5)$ & $-0.0003(4)$ \\
C10 & $0.0229(6)$ & $0.0231(6)$ & $0.0280(6)$ & $-0.0032(5)$ & $0.0040(5)$ & $0.0049(5)$ \\
C11 & $0.0394(8)$ & $0.0453(9)$ & $0.0415(8)$ & $-0.0018(7)$ & $0.0192(7)$ & $0.0115(7)$ \\
C12 & $0.0370(7)$ & $0.0264(6)$ & $0.0204(6)$ & $-0.0002(5)$ & $0.0024(5)$ & $-0.0014(5)$ \\
& & & & & & \\
\hline
\end{tabular}

Geometric parameters $\left(A,{ }^{\circ}\right)$

\begin{tabular}{|c|c|c|c|}
\hline $\mathrm{Si} 1-\mathrm{O} 2$ & $1.6435(9)$ & $\mathrm{C} 5-\mathrm{H} 5$ & 0.9500 \\
\hline $\mathrm{Si} 1-\mathrm{O}^{\mathrm{i}}$ & $1.6487(9)$ & C6-H6A & 0.9800 \\
\hline $\mathrm{Si} 1-\mathrm{C} 12$ & $1.8443(14)$ & $\mathrm{C} 6-\mathrm{H} 6 \mathrm{~B}$ & 0.9800 \\
\hline Sil-C11 & $1.8589(15)$ & C6- $\mathrm{H} 6 \mathrm{C}$ & 0.9800 \\
\hline $\mathrm{O} 1-\mathrm{C} 2$ & $1.3539(15)$ & $\mathrm{C} 7-\mathrm{H} 7$ & 0.9500 \\
\hline $\mathrm{O} 1-\mathrm{H} 1$ & $0.90(2)$ & $\mathrm{C} 8-\mathrm{H} 8 \mathrm{~A}$ & 0.9900 \\
\hline $\mathrm{O} 2-\mathrm{C} 8$ & $1.4345(14)$ & $\mathrm{C} 8-\mathrm{H} 8 \mathrm{~B}$ & 0.9900 \\
\hline $\mathrm{O} 3-\mathrm{C} 10$ & $1.4278(14)$ & $\mathrm{C} 9-\mathrm{C} 10$ & $1.5168(17)$ \\
\hline $\mathrm{N} 1-\mathrm{C} 1$ & $1.3343(18)$ & $\mathrm{C} 9-\mathrm{H} 9 \mathrm{~A}$ & 0.9900 \\
\hline $\mathrm{N} 1-\mathrm{C} 5$ & $1.3512(18)$ & C9-H9B & 0.9900 \\
\hline $\mathrm{N} 2-\mathrm{C} 7$ & $1.2808(16)$ & $\mathrm{C} 10-\mathrm{H} 10 \mathrm{~A}$ & 0.9900 \\
\hline $\mathrm{N} 2-\mathrm{C} 9$ & $1.4631(16)$ & $\mathrm{C} 10-\mathrm{H} 10 \mathrm{~B}$ & 0.9900 \\
\hline $\mathrm{C} 1-\mathrm{C} 2$ & $1.4143(18)$ & $\mathrm{C} 11-\mathrm{H} 11 \mathrm{~A}$ & 0.9800 \\
\hline $\mathrm{C} 1-\mathrm{C} 6$ & $1.5039(18)$ & $\mathrm{C} 11-\mathrm{H} 11 \mathrm{~B}$ & 0.9800 \\
\hline $\mathrm{C} 2-\mathrm{C} 3$ & $1.4041(17)$ & $\mathrm{C} 11-\mathrm{H} 11 \mathrm{C}$ & 0.9800 \\
\hline $\mathrm{C} 3-\mathrm{C} 4$ & $1.4147(17)$ & $\mathrm{C} 12-\mathrm{H} 12 \mathrm{~A}$ & 0.9800 \\
\hline $\mathrm{C} 3-\mathrm{C} 7$ & $1.4723(17)$ & $\mathrm{C} 12-\mathrm{H} 12 \mathrm{~B}$ & 0.9800 \\
\hline $\mathrm{C} 4-\mathrm{C} 5$ & $1.3832(18)$ & $\mathrm{C} 12-\mathrm{H} 12 \mathrm{C}$ & 0.9800 \\
\hline $\mathrm{C} 4-\mathrm{C} 8$ & $1.5085(17)$ & & \\
\hline $\mathrm{O} 2-\mathrm{Si} 1-\mathrm{O}^{\mathrm{i}}$ & $103.40(5)$ & $\mathrm{N} 2-\mathrm{C} 7-\mathrm{H} 7$ & 119.4 \\
\hline $\mathrm{O} 2-\mathrm{Si} 1-\mathrm{C} 12$ & $106.94(6)$ & $\mathrm{C} 3-\mathrm{C} 7-\mathrm{H} 7$ & 119.4 \\
\hline $\mathrm{O} 3{ }^{\mathrm{i}}-\mathrm{Si} 1-\mathrm{C} 12$ & $112.06(6)$ & $\mathrm{O} 2-\mathrm{C} 8-\mathrm{C} 4$ & $108.98(10)$ \\
\hline $\mathrm{O} 2-\mathrm{Si} 1-\mathrm{C} 11$ & $111.33(7)$ & $\mathrm{O} 2-\mathrm{C} 8-\mathrm{H} 8 \mathrm{~A}$ & 109.9 \\
\hline
\end{tabular}




\begin{tabular}{|c|c|c|c|}
\hline $\mathrm{O} 3^{\mathrm{i}}-\mathrm{Si} 1-\mathrm{C} 11$ & $109.52(6)$ & $\mathrm{C} 4-\mathrm{C} 8-\mathrm{H} 8 \mathrm{~A}$ & 109.9 \\
\hline $\mathrm{C} 12-\mathrm{Si} 1-\mathrm{C} 11$ & $113.16(7)$ & $\mathrm{O} 2-\mathrm{C} 8-\mathrm{H} 8 \mathrm{~B}$ & 109.9 \\
\hline $\mathrm{C} 2-\mathrm{O} 1-\mathrm{H} 1$ & $104.4(12)$ & $\mathrm{C} 4-\mathrm{C} 8-\mathrm{H} 8 \mathrm{~B}$ & 109.9 \\
\hline $\mathrm{C} 8-\mathrm{O} 2-\mathrm{Si} 1$ & $123.61(8)$ & $\mathrm{H} 8 \mathrm{~A}-\mathrm{C} 8-\mathrm{H} 8 \mathrm{~B}$ & 108.3 \\
\hline $\mathrm{C} 10-\mathrm{O} 3-\mathrm{Si}^{\mathrm{i}}$ & $123.50(8)$ & $\mathrm{N} 2-\mathrm{C} 9-\mathrm{C} 10$ & $110.90(10)$ \\
\hline $\mathrm{C} 1-\mathrm{N} 1-\mathrm{C} 5$ & $118.59(11)$ & $\mathrm{N} 2-\mathrm{C} 9-\mathrm{H} 9 \mathrm{~A}$ & 109.5 \\
\hline $\mathrm{C} 7-\mathrm{N} 2-\mathrm{C} 9$ & $118.05(11)$ & $\mathrm{C} 10-\mathrm{C} 9-\mathrm{H} 9 \mathrm{~A}$ & 109.5 \\
\hline $\mathrm{N} 1-\mathrm{C} 1-\mathrm{C} 2$ & $121.46(12)$ & N2-C9-H9B & 109.5 \\
\hline $\mathrm{N} 1-\mathrm{C} 1-\mathrm{C} 6$ & $118.23(12)$ & $\mathrm{C} 10-\mathrm{C} 9-\mathrm{H} 9 \mathrm{~B}$ & 109.5 \\
\hline $\mathrm{C} 2-\mathrm{C} 1-\mathrm{C} 6$ & $120.30(12)$ & $\mathrm{H} 9 \mathrm{~A}-\mathrm{C} 9-\mathrm{H} 9 \mathrm{~B}$ & 108.0 \\
\hline $\mathrm{O} 1-\mathrm{C} 2-\mathrm{C} 3$ & $122.14(11)$ & $\mathrm{O} 3-\mathrm{C} 10-\mathrm{C} 9$ & $109.10(10)$ \\
\hline $\mathrm{O} 1-\mathrm{C} 2-\mathrm{C} 1$ & $117.91(11)$ & $\mathrm{O} 3-\mathrm{C} 10-\mathrm{H} 10 \mathrm{~A}$ & 109.9 \\
\hline $\mathrm{C} 3-\mathrm{C} 2-\mathrm{C} 1$ & $119.92(11)$ & $\mathrm{C} 9-\mathrm{C} 10-\mathrm{H} 10 \mathrm{~A}$ & 109.9 \\
\hline $\mathrm{C} 2-\mathrm{C} 3-\mathrm{C} 4$ & $117.69(11)$ & $\mathrm{O} 3-\mathrm{C} 10-\mathrm{H} 10 \mathrm{~B}$ & 109.9 \\
\hline $\mathrm{C} 2-\mathrm{C} 3-\mathrm{C} 7$ & $120.63(11)$ & $\mathrm{C} 9-\mathrm{C} 10-\mathrm{H} 10 \mathrm{~B}$ & 109.9 \\
\hline $\mathrm{C} 4-\mathrm{C} 3-\mathrm{C} 7$ & $121.57(11)$ & $\mathrm{H} 10 \mathrm{~A}-\mathrm{C} 10-\mathrm{H} 10 \mathrm{~B}$ & 108.3 \\
\hline $\mathrm{C} 5-\mathrm{C} 4-\mathrm{C} 3$ & $118.12(12)$ & $\mathrm{Si} 1-\mathrm{C} 11-\mathrm{H} 11 \mathrm{~A}$ & 109.5 \\
\hline $\mathrm{C} 5-\mathrm{C} 4-\mathrm{C} 8$ & $119.45(11)$ & Sil-C11-H11B & 109.5 \\
\hline $\mathrm{C} 3-\mathrm{C} 4-\mathrm{C} 8$ & $122.36(11)$ & $\mathrm{H} 11 \mathrm{~A}-\mathrm{C} 11-\mathrm{H} 11 \mathrm{~B}$ & 109.5 \\
\hline $\mathrm{N} 1-\mathrm{C} 5-\mathrm{C} 4$ & $124.19(12)$ & $\mathrm{Si} 1-\mathrm{C} 11-\mathrm{H} 11 \mathrm{C}$ & 109.5 \\
\hline $\mathrm{N} 1-\mathrm{C} 5-\mathrm{H} 5$ & 117.9 & $\mathrm{H} 11 \mathrm{~A}-\mathrm{C} 11-\mathrm{H} 11 \mathrm{C}$ & 109.5 \\
\hline $\mathrm{C} 4-\mathrm{C} 5-\mathrm{H} 5$ & 117.9 & $\mathrm{H} 11 \mathrm{~B}-\mathrm{C} 11-\mathrm{H} 11 \mathrm{C}$ & 109.5 \\
\hline $\mathrm{C} 1-\mathrm{C} 6-\mathrm{H} 6 \mathrm{~A}$ & 109.5 & $\mathrm{Si} 1-\mathrm{C} 12-\mathrm{H} 12 \mathrm{~A}$ & 109.5 \\
\hline $\mathrm{C} 1-\mathrm{C} 6-\mathrm{H} 6 \mathrm{~B}$ & 109.5 & $\mathrm{Si} 1-\mathrm{C} 12-\mathrm{H} 12 \mathrm{~B}$ & 109.5 \\
\hline $\mathrm{H} 6 \mathrm{~A}-\mathrm{C} 6-\mathrm{H} 6 \mathrm{~B}$ & 109.5 & $\mathrm{H} 12 \mathrm{~A}-\mathrm{C} 12-\mathrm{H} 12 \mathrm{~B}$ & 109.5 \\
\hline $\mathrm{C} 1-\mathrm{C} 6-\mathrm{H} 6 \mathrm{C}$ & 109.5 & $\mathrm{Si} 1-\mathrm{C} 12-\mathrm{H} 12 \mathrm{C}$ & 109.5 \\
\hline $\mathrm{H} 6 \mathrm{~A}-\mathrm{C} 6-\mathrm{H} 6 \mathrm{C}$ & 109.5 & $\mathrm{H} 12 \mathrm{~A}-\mathrm{C} 12-\mathrm{H} 12 \mathrm{C}$ & 109.5 \\
\hline $\mathrm{H} 6 \mathrm{~B}-\mathrm{C} 6-\mathrm{H} 6 \mathrm{C}$ & 109.5 & $\mathrm{H} 12 \mathrm{~B}-\mathrm{C} 12-\mathrm{H} 12 \mathrm{C}$ & 109.5 \\
\hline $\mathrm{N} 2-\mathrm{C} 7-\mathrm{C} 3$ & $121.19(11)$ & & \\
\hline $\mathrm{O} 3-\mathrm{Si} 1-\mathrm{O} 2-\mathrm{C} 8$ & $68.47(10)$ & $\mathrm{C} 2-\mathrm{C} 3-\mathrm{C} 4-\mathrm{C} 8$ & $-176.10(11)$ \\
\hline $\mathrm{C} 12-\mathrm{Si} 1-\mathrm{O} 2-\mathrm{C} 8$ & $-173.10(9)$ & $\mathrm{C} 7-\mathrm{C} 3-\mathrm{C} 4-\mathrm{C} 8$ & $0.08(17)$ \\
\hline $\mathrm{C} 11-\mathrm{Si} 1-\mathrm{O} 2-\mathrm{C} 8$ & $-49.03(11)$ & $\mathrm{C} 1-\mathrm{N} 1-\mathrm{C} 5-\mathrm{C} 4$ & $0.17(19)$ \\
\hline $\mathrm{C} 5-\mathrm{N} 1-\mathrm{C} 1-\mathrm{C} 2$ & $1.61(18)$ & $\mathrm{C} 3-\mathrm{C} 4-\mathrm{C} 5-\mathrm{N} 1$ & $-1.42(19)$ \\
\hline $\mathrm{C} 5-\mathrm{N} 1-\mathrm{C} 1-\mathrm{C} 6$ & $-176.99(12)$ & $\mathrm{C} 8-\mathrm{C} 4-\mathrm{C} 5-\mathrm{N} 1$ & $175.64(12)$ \\
\hline $\mathrm{N} 1-\mathrm{C} 1-\mathrm{C} 2-\mathrm{O} 1$ & $179.60(11)$ & $\mathrm{C} 9-\mathrm{N} 2-\mathrm{C} 7-\mathrm{C} 3$ & $178.48(10)$ \\
\hline $\mathrm{C} 6-\mathrm{C} 1-\mathrm{C} 2-\mathrm{O} 1$ & $-1.83(17)$ & $\mathrm{C} 2-\mathrm{C} 3-\mathrm{C} 7-\mathrm{N} 2$ & $-3.14(17)$ \\
\hline $\mathrm{N} 1-\mathrm{C} 1-\mathrm{C} 2-\mathrm{C} 3$ & $-2.09(18)$ & $\mathrm{C} 4-\mathrm{C} 3-\mathrm{C} 7-\mathrm{N} 2$ & $-179.21(11)$ \\
\hline $\mathrm{C} 6-\mathrm{C} 1-\mathrm{C} 2-\mathrm{C} 3$ & $176.48(11)$ & $\mathrm{Si} 1-\mathrm{O} 2-\mathrm{C} 8-\mathrm{C} 4$ & $-154.64(8)$ \\
\hline $\mathrm{O} 1-\mathrm{C} 2-\mathrm{C} 3-\mathrm{C} 4$ & $179.01(11)$ & $\mathrm{C} 5-\mathrm{C} 4-\mathrm{C} 8-\mathrm{O} 2$ & $-106.34(13)$ \\
\hline $\mathrm{C} 1-\mathrm{C} 2-\mathrm{C} 3-\mathrm{C} 4$ & $0.78(17)$ & $\mathrm{C} 3-\mathrm{C} 4-\mathrm{C} 8-\mathrm{O} 2$ & $70.59(14)$ \\
\hline $\mathrm{O} 1-\mathrm{C} 2-\mathrm{C} 3-\mathrm{C} 7$ & $2.80(17)$ & $\mathrm{C} 7-\mathrm{N} 2-\mathrm{C} 9-\mathrm{C} 10$ & $108.51(12)$ \\
\hline $\mathrm{C} 1-\mathrm{C} 2-\mathrm{C} 3-\mathrm{C} 7$ & $-175.43(10)$ & $\mathrm{Si} 1^{\mathrm{i}}-\mathrm{O} 3-\mathrm{C} 10-\mathrm{C} 9$ & $143.92(9)$ \\
\hline $\mathrm{C} 2-\mathrm{C} 3-\mathrm{C} 4-\mathrm{C} 5$ & $0.87(16)$ & $\mathrm{N} 2-\mathrm{C} 9-\mathrm{C} 10-\mathrm{O} 3$ & $-65.04(13)$ \\
\hline $\mathrm{C} 7-\mathrm{C} 3-\mathrm{C} 4-\mathrm{C} 5$ & $177.05(11)$ & & \\
\hline
\end{tabular}

Symmetry code: (i) $-x+2, y,-z+1 / 2$. 
Hydrogen-bond geometry $\left(\AA,{ }^{\circ}\right)$

$C g 1$ is the centroid of the $\mathrm{N} 1 / \mathrm{C} 1-\mathrm{C} 5$ ring.

\begin{tabular}{lllll}
\hline$D-\mathrm{H} \cdots A$ & $D-\mathrm{H}$ & $\mathrm{H} \cdots A$ & $D \cdots A$ & $D-\mathrm{H} \cdots A$ \\
\hline $\mathrm{O} 1-\mathrm{H} 1 \cdots \mathrm{N} 2$ & $0.90(2)$ & $1.76(2)$ & $2.5923(15)$ & $153.2(18)$ \\
$\mathrm{C} 5-\mathrm{H} 5 \cdots \mathrm{O} 1^{\mathrm{ii}}$ & 0.95 & 2.69 & $3.5451(16)$ & 151 \\
$\mathrm{C} 6-\mathrm{H} 6 A \cdots \mathrm{O} 2^{\mathrm{iii}}$ & 0.98 & 2.59 & $3.3464(17)$ & 134 \\
$\mathrm{C} 7-\mathrm{H} 7 \cdots 3^{\mathrm{i}}$ & 0.95 & 2.57 & $3.4882(15)$ & 162 \\
$\mathrm{C} 9-\mathrm{H} 9 B \cdots \mathrm{O} 2^{\mathrm{iv}}$ & 0.99 & 2.60 & $3.5087(16)$ & 153 \\
$\mathrm{C} 9-\mathrm{H} 9 B \cdots C g 1^{\mathrm{iv}}$ & 0.99 & 3.31 & $4.039(2)$ & 131 \\
$\mathrm{C} 11-\mathrm{H} 11 A \cdots C g 1^{\mathrm{ii}}$ & 0.98 & 2.85 & $3.7880(2)$ & 160 \\
\hline
\end{tabular}

Symmetry codes: (i) $-x+2, y,-z+1 / 2$; (ii) $x,-y+1, z-1 / 2$; (iii) $x,-y+1, z+1 / 2$; (iv) $-x+3 / 2,-y+1 / 2,-z+1 / 2$. 